\title{
Histoplasma panniculitis in a patient with systemic lupus erythematosus*
}

\author{
Luis Rodrigo Flores-Bozo ${ }^{1}$, Edgar Ortiz-Brizuela², Luis Enrique Soto-Ramírez²
}

DOI: http:/ / dx.doi.org/10.1590/abd1806-4841.20197636

\begin{abstract}
Histoplasmosis usually presents primarily as lung infection. Occasionally, mainly in immunocompromised hosts, it can spread and cause systemic manifestations. Skin lesions have been reported in 10 to 15 percent of cases of disseminated histoplasmosis, and panniculitis has been described as an unusual form of presentation in affected patients. We present the case of a patient with systemic lupus erythematosus who presented cellulitis due to disseminated histoplasmosis.
\end{abstract}

Keywords: Histoplasma; Lupus erythematosus, systemic; Panniculitis

A 48-year-old woman with systemic erythematosus lupus presented with a six-month history of ill-defined, painful, erythematous, edematous plaques on the left thigh and leg. She was on treatment with prednisone $5 \mathrm{mg} /$ day and mycophenolate mofetil $4 \mathrm{~g} /$ day. Stains and cultures for bacteria, mycobacteria, and fungi from a skin biopsy were negative. Pathological examination revealed septal and lobular panniculitis. Lupus panniculitis was suspected and prednisone $30 \mathrm{mg} /$ day was prescribed, without improvement. One month later she presented with fever and the erythema worsened; piperacillin-tazobactam plus vancomycin were commenced. A new set of skin and blood cultures and a Histoplasma capsulatum urinary antigen were negative; the pathological examination revealed cutaneous mucinosis.

Subsequently, the dermatosis progressed, and she developed haemophagocytic syndrome (Figure 1). A third skin biopsy revealed septal and lobular panniculitis with oval and narrow-based budding yeasts observed with the PAS staining (Figure 2). White mold colonies grew in the Sabouraud agar media from the skin and bone marrow samples; the direct examination with cotton-blue staining showed septate, hyaline hyphae, and tuberculate macroconidia compatible with Histoplasma capsulatum (Figure 3). Liposomal amphotericin B was commenced with improvement of the skin lesions. Unfortunately, she developed nosocomial pneumonia and died a few weeks later.

Histoplasmosis is a common endemic mycosis that is usually asymptomatic but occasionally results in severe illness. Histoplasmosis and its causative agent, Histoplasma capsulatum, are found worldwide but particularly in North and Central America. Histoplasmosis usually presents primarily as lung infection, and occasionally, mainly in immunocompromised hosts, it can spread and cause systemic manifestations. Among the endemic mycoses, it is the most common cause for hospitalization. ${ }^{1}$ Histoplasmosis is a major cause of opportunistic infection in several Latin American countries. ${ }^{2}$ Skin lesions have been reported in 10 to 15 percent of

\footnotetext{
Received 12 September 2017.

Accepted 14 January 2018.

* Work conducted at the Instituto Nacional de Ciencias Médicas y Nutrición Salvador Zubirán, Mexico City, Mexico.

Financial support: None.

Conflict of interest: None.

Department of Dermatology, Hospital Instituto Nacional de Ciencias Médicas y Nutrición Salvador Zubirán, Mexico City, Mexico.

Department of Infectious Diseases, Hospital Instituto Nacional de Ciencias Médicas y Nutrición Salvador Zubirán, Mexico City, Mexico.
}

\section{MAILING AdDRESS:}

Luis Enrique Soto-Ramirez

E-mail: lesoto@hotmail.com 


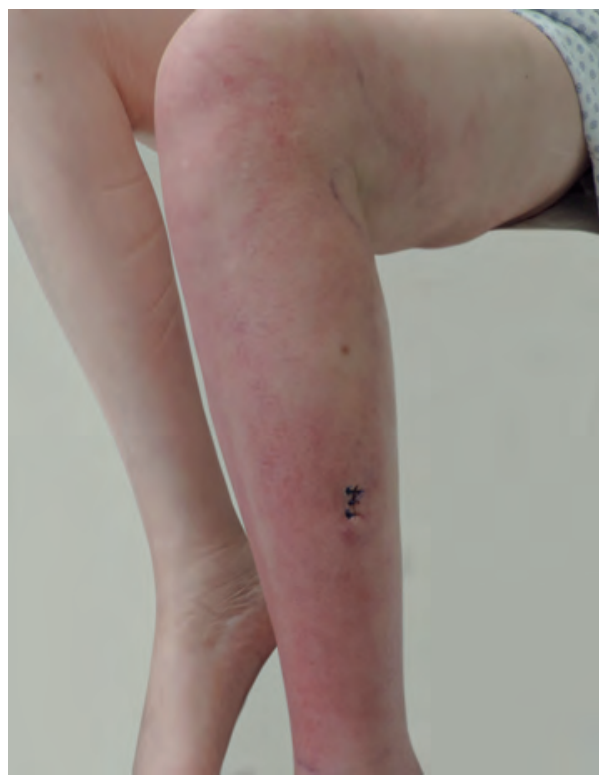

Figure 1: Ill-defined, painful, erythematous and edematous plaques on the left thigh and leg

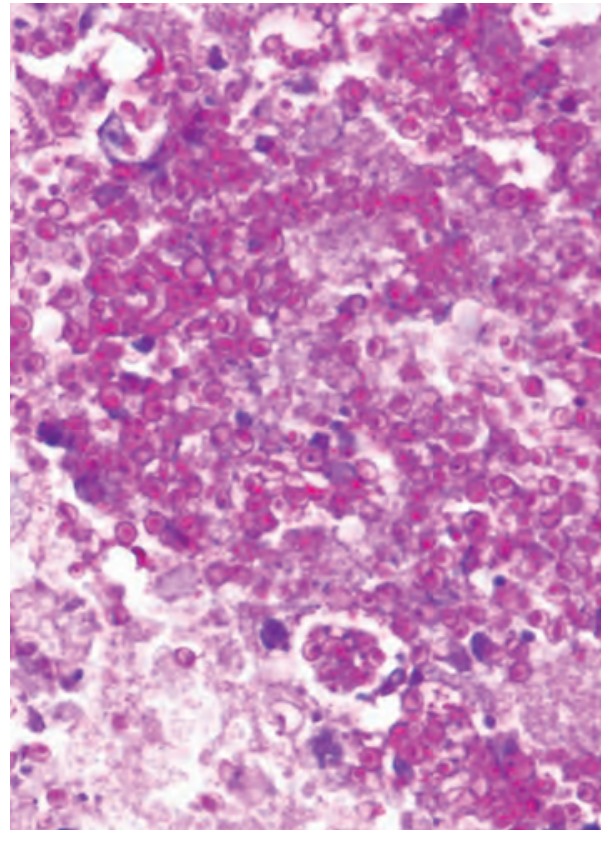

FigURE 2: Skin

biopsy. PAS staining showing oval yeasts (2-to $4 \mu \mathrm{m})$, some with narrow-based budding $(x 100)$

cases of disseminated histoplasmosis. It has been described that the skin involvement is considerably more frequent in immunosuppressed patients, ${ }^{3}$ especially in patients living with HIV. The characteristic lesions include nodules, papules, plaques, ulcers, vesicles, pustules, abscesses, and generalized dermatitis. ${ }^{3,4}$

Panniculitis is an inflammatory disease of the subcutaneous fat that can be secondary to a broad spectrum of causes (e.g. infection, external insults, malignancy). It is a relatively uncommon con-
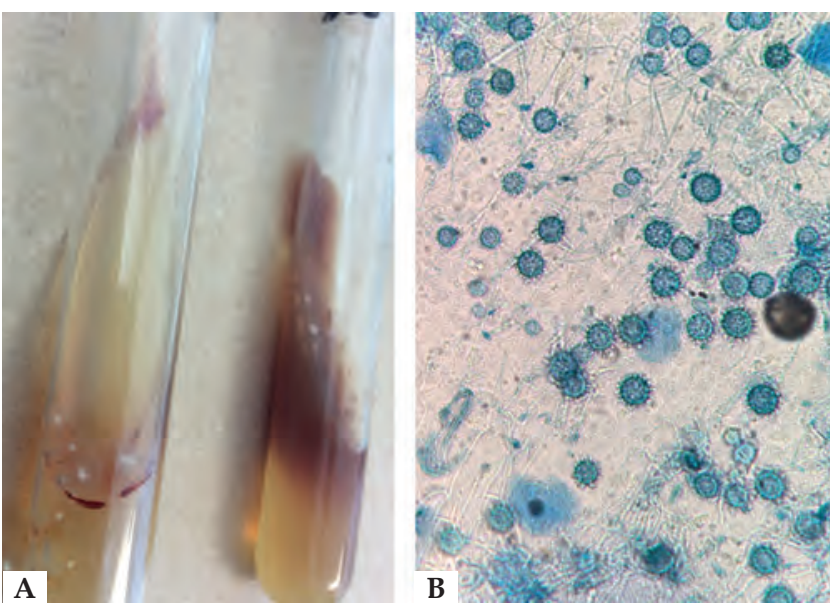

Figure 3: A - Sabouraud dextrose agar culture at $25^{\circ} \mathrm{C}$ showing white, cotton-like colonies of Histoplasma capsulatum. B - Hyaline and septate hyphae with abundant tuberculate, thick-walled macroconidia (Lactophenol cotton blue stain $\times 40$ )

dition that usually presents with inflammatory nodules or plaques. ${ }^{5}$ Given the non-specific clinical presentation of the panniculitides, the clinician must have a high index of suspicion to exclude an infectious etiology based on the patients' risk factors and the associated clinical manifestations.

Panniculitis has been described as an unusual form of presentation in histoplasmosis. ${ }^{6,7}$ In two studies, stains for fungi performed on skin biopsy specimens revealed the organism in 86 to 100 percent of cases of HIV-associated disseminated histoplasmosis. ${ }^{3,8}$ Classically, organisms are observed intracellularly, however, they may primarily be seen extracellularly, making it more difficult to suspect the diagnosis. ${ }^{9}$ Granulomas are uncommon; more characteristic is the presence of an infiltrate composed of macrophages full of H. capsulatum yeasts. ${ }^{9}$ These lesions respond well to therapy, usually resolving within a few weeks. ${ }^{8}$

Lupus panniculitis was initially suspected based on SLE history, histological findings in the first and second skin biopsy and in the absence of microbiological isolates; however, the immunosuppression caused by an increase in the dose of prednisone generated progression of the dermatosis and clinical deterioration, despite broad-spectrum antibiotic treatment which was initiated because of the suspected infectious etiology. All this led to a third biopsy and skin culture, where finally the infectious agent was documented and the antifungal treatment started. As previously reported, histoplasmosis could be misdiagnosed as a consequence of connective tissue diseases. ${ }^{10}$

The evolution in this clinical case is relevant and reinforces the need to include fungal agents in the differential diagnosis of panniculitis in the context of immunosuppressed patients. $\square$

\section{ACKNOWLEDGEMENTS}

We thank Dr. Josune Echevarría Keel and BSc Andrea Rangel Cordero for their contribution in care of this patient and providing some images for this manuscript. 


\section{REFERENCES}

1. Chu JH, Feudtner C, Heydon K, Walsh TJ, Zaoutis TE. Hospitalizations for endemic mycoses: a population-based national study. Clin Infect Dis. 2006;42:822-5.

2. Colombo AL, Tobón A, Restrepo A, Queiroz-Telles F, Nucci M. Epidemiology of endemic systemic fungal infections in Latin America. Med Mycol. 2011;49:785-98.

3. Assi MA, Sandid MS, Baddour LM, Roberts GD, Walker RC. Systemic histoplasmosis: a 15-year retrospective institutional review of 111 patients. Medicine (Baltimore). 2007;86:162-9.

4. Chang P, Rodas C. Skin lesions in histoplasmosis. Clin Dermatol. 2012;30:592-8

5. Patterson JW. Panniculitis. In: Bolognia JL, Jorizzo JL, Schaffer JV, editors. Dermatology. London: Elsevier Saunders; 2012. p. 1641-62.

6. McGuinn ML, Lawrence ME, Proia L, Segreti J. Progressive Disseminated Histoplasmosis Presenting as Cellulitis in a Renal Transplant Recipient. Transplant Proc. 2005;37:4313-4.
7. Marques SA, Hozumi S, Camargo RM, Carvalho MF, Marques ME. Histoplasmosis presenting as cellulitis 18 years after renal transplantation. Med Mycol. 2008;46:725-8

8. Cohen PR, Bank DE, Silvers DN, Grossman ME. Cutaneous lesions of disseminated histoplasmosis in human immunodeficiency virus-infected patients. J Am Acad Dermatol. 1990;23:422-8.

9. Eidbo J, Sanchez RL, Tschen JA, Ellner KM. Cutaneous manifestations of histoplasmosis in the acquired immune deficiency syndrome. Am J Surg Pathol. 1993;17:110-6.

10. Ceccato F, Gongora V, Zunino A, Roverano S, Paira S. Unusual manifestation of histoplasmosis in connective tissue diseases. Clin Rheumatol. 2007;26:1717-9.

\section{Luis Rodrigo Flores-Bozo}

Approval of the final version of the manuscript; Elaboration and writing of the manuscript; Critical review of the literature; Critical review of the manuscript

Edgar Ortiz-Brizuela

(iD) ORCID 0000-0001-7169-8459

Approval of the final version of the manuscript, Conception and planning of the study, Elaboration and writing of the manuscript, Obtaining, analyzing and interpreting the data, Effective participation in research orientation, Intellectual participation in propaedeutic and/or therapeutic conduct of the cases studied, Critical review of the manuscript

Luis Enrique Soto-Ramírez

(iD) ORCID 0000-0001-5809-3121

Approval of the final version of the manuscript; Elaboration and writing of the manuscript; Critical review of the literature; Critical review of the manuscript

How to cite this article: Flores-Bozo LR, Ortiz-Brizuela E, Soto-Ramírez LE. Histoplasma panniculitis in a patient with systemic lupus erythematosus. An Bras Dermatol. 2019;94(1):96-8. 